\title{
Association between Serum Levels of Vitamin D, Vitamin B12 and Folate with Oxidative Biomarkers in Diabetic Type 2 Patients in People in Erbil City: A Case-Control Study Gulzar Ismael Ibrahim (PhD) ${ }^{1}$, Hemn Jameel Majeed (MSc) ${ }^{2}$, Pshtiwan Abdullah Yousif $(\mathrm{MSc})^{3}$ and Saman Muhsin Abdulkareem $(\mathrm{PhD})^{4}$
}

\begin{abstract}
Background: Diabetes mellitus is the largest endocrine disorder in the world that refers to chronic hyperglycemia caused by impairment in both insulin secretion and action. Folate, vitamin B12, and vitamin D deficiency are related to the prevalence of type 2 diabetes mellitus.

Objective: This study has been carried out to investigate the role of folate, vitamins B12, and $\mathrm{D}$ status during the progression of diabetic type 2 patients compared to non-diabetic control subjects.
\end{abstract}

Patients and Methods: We measured the plasma levels of folate, vitamins B12, and D, along with blood glucose, $\mathrm{HbA}_{1 \mathrm{c}}$, and oxidative biomarkers in 50 diabetic type 2 patients and 50 healthy subjects used as a control group.

Results: The findings revealed that the serum level of malondialdehyde (MDA) significantly increased in diabetic patients compared to healthy subjects, and the serum level of nitric oxide (NO) in T2D patients lower than in control. The concentration of vitamin D, B12, and folate in T2D patients significantly lower than non-T2D control. The results also showed a significant relationship between serum vitamin $\mathrm{D}$, MDA, and $\mathrm{NO}$ with $\mathrm{HbA}_{1 \mathrm{c}}$, while there was an insignificant correlation between vitamin $\mathrm{B} 12$ and folate with $\mathrm{HbA}_{1 \mathrm{c}}$.

Conclusion: The oxidative biomarkers and vitamins D, B12, and folate level can play an important role in the development and progression of T2D.

Keywords: Oxidative Stress, Vitamin D, Vitamin B12, Folic acid, type 2 diabetes Corresponding Author: gulzar.ibrahim@su.edu.krd

Received: $15^{\text {th }}$ September 2020

Accepted: $3^{\text {rd }}$ November 2020

DOI:https://doi.org/10.26505/DJM.19025660915

\footnotetext{
${ }^{1,2,3}$ Department of Chemistry - College of Education - Salahaddin University-Erbil- Iraq

${ }^{4}$ Department of Biology - College of Education - Salahaddin University- Erbil- Iraq
}

\section{Introduction}

Diabetes mellitus is the largest endocrine disorder in the world that refers to chronic hyperglycemia caused by impairment in both insulin secretion and action. Type 2 diabetes mellitus (T2D) is characterized by elevated blood sugar and circulating lipids, along with insulin resistance [1]. In this case, the metabolism of carbohydrates, fats, and 
proteins is disrupted and leads to lifethreatening health complications. Patients with diabetes have high mortality due to multiple complications from insulin deficiency or lack of insulin. The World Health Organization stated in 2012 that diabetes directly causes 1.5 million deaths and indirectly is responsible for the deaths of 2.2 million people because of increased cardiovascular risks and other diseases. Also, in 2014, 422 million individuals worldwide have diabetes and had a prevalence of $8.5 \%$ among adults. The highest deaths (1.5 million) from hyperglycemia happen in middle-income countries and the lowest $(0.3$ million) in low-income countries [2].

Many factors, such as oxidative stress, are involved in the development of diabetes and its problems, which are well known. Oxidative stress refers to increased production of free radicals or weakened antioxidant defense mechanisms [3]. Hyperinsulinaemia and hyperglycemia enhance the manufacture of free radicals and induce oxidative damage. Increased levels of free radicals impaired insulin secretion and induce insulin resistance and play a critical role in diabetic complications, including macro micro-vascular damage [4]. Factors such as levels of glutathione (GSH), malondialdehyde (MDA), and nitric oxide (NO) are measured to assess oxidative stress status. Studies have also shown that endogenous antioxidant levels suchlike vitamins $\mathrm{A}, \mathrm{C}, \mathrm{E}$ and $\mathrm{D}$, and other vitamins are significantly reduced in diabetic patients and can be a risk factor for diabetes complications [5, 6]. Vitamin D is a group of fatty acids soluble steroids that have several biological effects. It was reported that an adequate level of vitamin $\mathrm{D}$, as well as its classic role in calcium and phosphorus homeostasis and bone remodeling, can enhance immune system function.

On the other hand, studies have revealed that vitamin D deficiency can contribute to the prevalence of T2D [7]. Other studies have shown that adequate serum levels of vitamin D can play an important role in insulin release and insulin sensitivity [8]. In addition, vitamin D deficiency altered the glucose homeostasis and has a negative relationship with glycated hemoglobin $\left(\mathrm{HbA}_{1 \mathrm{c}}\right)$ levels in gestational diabetes[9]. Folic acid, folate, or B9 is an important cofactor for transamination from homocysteine to methionine. Hence, folate deficiency results in hyperhomocysteinemia, which is a biomarker for heart health.

In addition to hyperhomocysteinemia, folate deficiency can be involved in chronic complications in diabetic patients [10]. Moreover, it was stated that levels of folic acid in plasma and erythrocytes were reduced in patients with T2D [11]. Otherwise, clinical studies have shown that serum folate amounts in patients with T2D were greater than in healthy subjects [12]. Assessment of vitamin B12 or cobalamin and its deficiency in patients with T2D is of clinical importance. It can be referred to as peripheral neuropathy [13]. However, the relationship between these vitamins and oxidative stress biomarkers is not perfectly understood. Therefore, this study aimed to assess the serum levels of oxidative stress biomarkers and levels of some vitamins in T2D patients 
and healthy controls, and their correlation with $\mathrm{HbA}_{1 \mathrm{c}}$.

\section{Patients and Methods}

Anthropometric data and sample preparation: In this case-control study, 50 T2D patients (case group) were compared with 50 healthy subjects (control group) in terms of serum fasting and random blood sugar (FBS, RBS), $\mathrm{HbA}_{1 \mathrm{c}}$, and oxidative biomarkers levels. This was conducted in Razgari hospital, Erbil. Medical history, demographics, and lifestyle characteristics were collected using standard questionnaires. Body mass index (BMI) was measured as body weight $(\mathrm{kg})$ divided by the square of the person's height $\left(\mathrm{m}^{2}\right)$. Education status was classified as high school or lower and high school or higher. T2D patients between the age group of 40-70 years of either sex with 5 years history of diabetes were recruited from the outpatient department of medicine. Healthy subjects between the age group of 40-70 years of either sex were enrolled as a control group. Patients and controls voluntarily participated in the study. In this study, we excluded patients suffering from inflammation and chronic infection, gestational diabetes, type 1 diabetes, renal disease, liver disease, and thalassemia, as well as those treated with metformin, diuretics, antioxidants, and steroids, or consumed alcohol or cigarettes. Informed consent was obtained from participants. The research protocol was approved by the research ethics committee of the Department of Biology, College of Education of Salahaddin University-Erbil.
Laboratory measurements: Fasting blood sugar (FBS), random blood sugar (RBS), $\mathrm{HbA}_{1 \mathrm{c}}$, serum NO levels, serum MDA level, and vitamins (D, B12, and folate) were measured in all the participants. The glucose oxidase method was used to measure blood glucose [14]. Serum NO concentration was measured by the Griess method [15]. The serum concentration of MDA was measured as a marker for lipid peroxidation [16]. The level of serum $\mathrm{HbA}_{1 \mathrm{c}}$ was evaluated by the resin binding method [17]. Serum levels of $25(\mathrm{OH}) \mathrm{D}$ were determined through the radioimmunoassay (RIA) technique by using 25(OH)D125 I RIA kit (DiaSorin, USA). The Plasma level of folate and vitamin B12 was evaluated by commercially available kits (Roche) according to company protocol instructions using the Cobas Integra 400-plus system.

\section{Statistical analysis}

The data were stated as mean \pm standard deviation (SD). An independent t-test was used to compare the patient group with the control group. Statistical significance was set at $\mathrm{P} \leq 0.05$.

\section{Results}

Table (1) indicates the characteristics of the participants in this study. In this study, FBS and $\mathrm{HbA}_{1 \mathrm{c}}$ levels were used to diagnose and monitor diabetes. The results showed that FBS and $\mathrm{HbA}_{1 \mathrm{c}}$ significantly were higher in persons with diabetes than in healthy individual control. There was no significant variance in $\mathrm{FBS}$ and $\mathrm{HbA}_{1 \mathrm{c}}$ between men and women with diabetes. 
Table (1): The mean $( \pm \mathrm{SD})$ value of FBS and HbA1c in diabetic and healthy subjects

\begin{tabular}{|c|c|c|c|c|}
\hline Subject & Male control & DM (Male) & Female control & DM (Female) \\
\hline Number of participants & 25 & 25 & 25 & 25 \\
\hline Age (year) & $52 \pm 6.3$ & $54 \pm 5.4$ & $53 \pm 5.6$ & $54 \pm 6.5$ \\
\hline Body mass index $\left(\mathrm{Kg} / \mathrm{m}^{2}\right)$ & $23.3 \pm 2.3$ & $32.3 \pm 3.1^{* * *}$ & $25.1 \pm 2.6$ & $31 \pm 4.41^{* * *}$ \\
\hline HbA1c \% & $5.42 \pm 0.58$ & $8.97 \pm 1.14^{\text {**** }}$ & $5.40 \pm 0.64$ & $8.47 \pm 1.44^{* * * *}$ \\
\hline FBS (mg/dL) & $92 \pm 11.4$ & $208 \pm 27.1^{* * * *}$ & $95 \pm 31.2$ & $201 \pm 12.3^{\text {**** }}$ \\
\hline RBS (mg/dL) & $95.10 \pm 8.44$ & 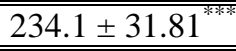 & $100.2 \pm 7.4$ & $246.6 \pm 34.51^{* * *}$ \\
\hline
\end{tabular}

*Significant $(\mathrm{P}<0.05)$, **highly significant $(\mathrm{P}<0.01)$, ***very highly significant $(\mathrm{P}<0.001)$

The mean values $( \pm \mathrm{SD})$ of serum MDA and

NO levels are presented in Table (2). The results indicated that the level of MDA in patients with diabetes was meaningfully higher than the control group; the level of NO in T2D patients was significantly less than the control group; there was insignificant variance in serum MDA and NO levels between men and women with diabetes.

Table (2): The mean values $( \pm \mathrm{SD})$ of serum oxidative parameters between diabetic and control subjects

\begin{tabular}{|c|c|c|c|c|}
\hline Subject & Male control & T2D (Male) & Female control & T2D (Female) \\
\hline Number of participants & 25 & 25 & 25 & 25 \\
\hline MDA (nmol /mL) & $1.95 \pm 0.75$ & $3.49 \pm 0.92^{*}$ & $2.65 \pm 0.12$ & $4.11 \pm 0.36^{*}$ \\
\hline $\mathrm{NO}(\mu \mathrm{mol} / \mathrm{L})$ & $39.21 \pm 3.17$ & $17.41 \pm 8.7^{*}$ & $45.67 \pm 3.41$ & $19.51 \pm 7.3^{*}$ \\
\hline
\end{tabular}

The mean values $( \pm \mathrm{SD})$ of serum vitamins results also displayed that the level of B12, folate, and D3 are presented in Table 3. vitamin $D$ in T2D patients was not The results indicated that the level of these significantly differed from the case-control. vitamins in patients with diabetes was Healthy men and men with diabetes had significantly lesser than the case-control; the vitamin deficiency.

Table (3): The mean values $( \pm \mathrm{SD})$ of some vitamins between diabetic and control subjects

\begin{tabular}{|l|c|c|c|c||}
\hline \hline Groups & Female-Control & Female-Patients & Male-Control & Male-Patients \\
\hline \hline Vitamin D (ng/mL) & $28.44 \pm 6.86$ & $12.75 \pm 7.88^{* * * *}$ & $13.73 \pm 2.54$ & $11.96 \pm 6.29$ \\
\hline \hline Folic acid (ng/mL) & $15.87 \pm 5.04$ & $7.89 \pm 2.74^{* * *}$ & $13.08 \pm 4.98$ & $6.55 \pm 9.9^{* * *}$ \\
\hline \hline Vitamin B12 pg/mL & $711.04 \pm 57.28$ & $592.01 \pm 46.47^{* * *}$ & $556.45 \pm 27.06$ & $416.09 \pm 34.61^{* * *}$ \\
\hline
\end{tabular}

*Significant $(\mathrm{P}<0.05), * *$ highly significant $(\mathrm{P}<0.01), * * *$ very highly significant $(\mathrm{P}<0.001)$

\section{Discussion}

T2D is a progressive disease marked by high amounts of circulating glucose and lipids along with insulin resistance. In this study, we measured HbA1c levels as well as blood glucose. The level of these factors significantly increased in patients with T2D. Increased glucose levels are common in patients with T2D due to impaired insulin function and have been well documented by many studies. The pathogenesis of T2D is multifactorial. Animal and clinical studies showed that oxidative stress is one of the causes of T2D and its complications. In agreement with these findings, this study's outcomes revealed that MDA and NO significantly increased and decreased respectively, in patients with $\mathrm{T} 2 \mathrm{D}$ compared to control. Results also demonstrated that 
there is no important difference between female and male diabetic patients and nondiabetic control. MDA is a byproduct of lipid peroxidation that increased in obesity, metabolic syndrome, and T2D patients. 30$35 \%$ of glucose is metabolized by the polyol pathway in T2D conditions. Under these conditions, vascular endothelial cell function is impaired, and NO production is reduced. Hyperinsulinemia and hyperglycemia are the main causes of free radical production and oxidative damage in patients with T2D. Increased free radical production leads to dyslipidemia, beta-cell dysfunction, and insulin resistance [18].

On the other hand, as we know, there is a positive relationship between blood glucose and $\mathrm{HbA}_{1 \mathrm{c}} \cdot \mathrm{HbA}_{1 \mathrm{c}}$ is an important biomarker for evaluating chronic blood glucose concentration and diagnosing diabetes. Increasing its plasma level leads to aggregation of blood cells and increases blood viscosity, resulting in impaired blood flow that could be one of the causes of high blood pressure in diabetes.

The results also exhibited a considerable correlation between serum MDA and NO with $\mathrm{HbA}_{1 \mathrm{c}}$; it seems reasonable because hyperglycemia causes oxidative stress. The study outcomes also showed that the serum vitamin D level in female patients with diabetes significantly decreased compared to that in non- diabetic control. In agreement with our findings, Calvo-Romero and Ramiro-Lozano (2015) stated that "vitamin D deficiency is common in type 2 diabetic patients. There are inverse correlations between vitamin $\mathrm{D}$ and metabolic control and insulin resistance" [19].
Moreover, in a study that was conducted by Saif-Elnasr et al (2017), they stated that "low level of vitamin D might play a significant role in T2D pathogenesis and this finding may have therapeutic implications as cautious vitamin D supplementation may improve glycemic control and oxidative stress in T2D" [20].

The results also displayed that male patients with or without diabetes were deficient in vitamin $\mathrm{D}$ and did not show a significant difference, despite a decrease in the diabetic group. Vitamin D deficiency is a common problem in developing countries and is responsible for the incidence of numerous diseases. Vitamin D deficiency in these people can be attributed to malnutrition and lack of proper nutrition and health. Some authors have reported an association among air pollution, industrial lifestyle, and low intake of seafood with low levels of serum vitamin D. Air pollution may play a role in the highest rate of vitamin D deficiency [21]; further investigations are required in this field to confirm this issue.

Our findings also showed that there is a significant negative correlation between serum vitamin $\mathrm{D}$ levels and $\mathrm{HbA}_{1 \mathrm{c}}$. This negative association could be due to the role of vitamin $\mathrm{D}$ in the control of blood sugar in normal conditions, which in diabetic patients is impaired by vitamin $\mathrm{D}$ deficiency. In addition to participating in calcium and phosphorus homeostasis and bone remodeling, vitamin $\mathrm{D}$ is also involved in $\beta$ cell function, insulin release, and insulin sensitivity. 1,25(OH)2D, biological form of vitamin $\mathrm{D}$, in beta cells enhances insulin production and secretion. Studies have also 
shown that vitamin D possess antioxidant power. On the other hand, vitamin D supplements can also recover oxidative stress in patients with diabetes by improving glucose regulation.

The data of this study showed that patients with diabetes have deficient in folic acid, and there was an insignificant variance between males and females with diabetes. These results are similar to the results of Malaguarnera, Gagliano [11]. They informed that the level of folic acid in plasma and erythrocytes decreased in patients with T2D. Plasma and erythrocyte folate levels have been broadly accepted as direct biochemical indicators of folate status. So we have chosen plasma folate because it is known as a direct pointer of folate in the body. Although we excluded patients using metformin, it was reported that it decreased serum folate levels [22]. Folate deficiency is connected with cardiovascular disease, anemia, hyperhomocysteinemia, DNA damage, and impaired NO production. Moreover, it was reported that homocysteine and diabetes enhance oxidative stress and decrease NO formation and may cause endothelial dysfunction [23, 24]. Although it appears that the decrease in NO in diabetic patients may also be due to Folate deficiency, further studies are needed.

The results of this study showed that the level of vitamin B12 significantly decreased in patients with diabetes. The results also showed that there is a significant alteration between the levels of this vitamin in healthy men and women with diabetes. Many studies have shown vitamin B12 deficiency among patients with type 1 and type 2 Diabetes [25].
In a study conducted by Qureshi, Ainsworth [26], they considered vitamin 12 deficiency to be less than $150 \mathrm{pg} / \mathrm{ml}$ in serum and showed that $33 \%$ of diabetic patients had vitamin 12 deficiency. Currently, no published guidelines are supporting routine screening for vitamin B12 deficiency in T2D patients. However, it is recommended that a blood vitamin B12 assessment be performed before metformin treatment is initiated [25]. Because many studies agree that taking metformin in patients with T2D causes vitamin B12 deficiency [27, 28]. Therefore, definitive conclusions in this regard should be exercised with caution, although some explanations have been suggested to reduce these. In a cross-sectional case-control study conducted by Satyanarayana, Balakrishna [29], they showed that the level of folic acid, B6, and B12 in patients with diabetes significantly lower than case-control. They also showed a significant relationship between hyperhomocysteinemia and diabetes. In addition, they showed that vitamin B12 levels were not affected by age, BMI, or duration of diabetes because the association between vitamin B12 and these variables was not statistically significant. On the other hand, we know that hypohomocysteinemia has a variety of causes, including dietary deficiencies of folic acid and vitamins B6 and B12 [29, 30]. Therefore, vitamin B12 deficiency in people with diabetes seems to be partly due to the lack of adequate sources of this vitamin and more studies are needed.

\section{Conclusions}

The results demonstrated that lowering the levels of vitamin D, B12, and folate can be 
the risk factors for type 2 Diabetes in the urban people, and further studies are needed to introduce them as pathogenesis of diabetes.

\section{References}

[1]American Diabetes A. Diagnosis and classification of diabetes mellitus. Diabetes Care. 2009; 32 Suppl 1(Suppl 1): S62-S7.

[2]World Health O. Global report on diabetes: World Health Organization. Report No: 9789241565257 WHO. 2016.

[3]Ibrahim GI, Abdulkareem SM, Hasan LM. Estimation of Nitric Oxide, Malondialdehyde, and Adenosine Deaminase in Serum of Hypertensive Patients and Normotensive Individuals in Erbil City. Tikrit Journal of Pure Science. 2019; 24(3):17-22.

[4]Jelodar G, Mohammadi M, Akbari A, Nazifi S. Cyclohexane extract of walnut leaves improves indices of oxidative stress, total homocysteine and lipids profiles in streptozotocin-induced diabetic rats. Physiological Reports. 2020; 8(1):e14348.

[5]Akbari A. An overview of the characteristics and function of vitamin $\mathrm{C}$ in various tissues: relying on its antioxidant function. Zahedan Journal of Research in Medical Sciences. 2016; 18(11): e4037.

[6] Oguntibeju OO. Type 2 diabetes mellitus, oxidative stress and inflammation: examining the links. International Journal of Physiology, Pathophysiology and Pharmacology. 2019; 11(3):45-63.

[7]Valdés-Ramos R, Ana Laura G-L, Beatriz Elina M-C, Alejandra Donaji B-A. Vitamins and type 2 diabetes mellitus. Endocrine, Metabolic \& Immune Disorders-Drug Targets (Formerly Current Drug Targets-
Immune, Endocrine \& Metabolic Disorders). 2015; 15(1):54-63.

[8]Alvarez JA, Ashraf A. Role of vitamin D in insulin secretion and insulin sensitivity for glucose homeostasis. International Journal of Endocrinology. 2010; 2010: 351385.

[9]Sung CC, Liao MT, Lu KC, Wu CC. Role of vitamin $\mathrm{D}$ in insulin resistance. Journal of Biomedicine and Biotechnology. 2012; 2012: $1-11$.

[10]Wulffele MG, Kooy A, Lehert P, Bets D, Ogterop JC, Borger Van Der Burg B, et al. Effects of short-term treatment with metformin on serum concentrations of homocysteine, folate and vitamin B12 in type 2 diabetes mellitus: a randomized, placebocontrolled trial. Journal of Internal Medicine. 2003; 254(5): 455-63.

[11]Malaguarnera G, Gagliano C, Salomone S, Giordano M, Bucolo C, Pappalardo A, et al. Folate status in type 2 diabetic patients with and without retinopathy. Clinical Ophthalmology. 2015; 9:1437-42.

[12]Bartlett HE, Eperjesi F. Nutritional supplementation for type 2 diabetes: a systematic review. Ophthalmic and Physiological Optics. 2008; 28(6):503-23. [13]Obeid R, Jung J, Falk J, Herrmann W, Geisel J, Friesenhahn-Ochs B, et al. Serum vitamin B12 not reflecting vitamin B12 status in patients with type 2 diabetes. Biochimie. 2013; 95(5):1056-61.

[14]Saifer A, Gerstenfeld S. The photometric microdetermination of blood glucose with glucose oxidase. The Journal of Laboratory and Clinical Medicine. 1958; 51(3):448-60. [15]Miranda KM, Espey MG, Wink DA. A rapid, simple spectrophotometric method for 
simultaneous detection of nitrate and nitrite. Nitric Oxide. 2001; 5(1):62-71.

[16] Bhutia Y, Ghosh A, Sherpa ML, Pal R, Mohanta PK. Serum malondialdehyde level: Surrogate stress marker in the Sikkimese diabetics. Journal of Natural Science, Biology, and Medicine. 2011; 2(1):107.

[17]Weykamp C, John WG, Mosca A. A review of the challenge in measuring hemoglobin A1c. Journal of Diabetes Science and Technology. 2009; 3(3):439-45.

[18]Ostovar M, Akbari A, Anbardar MH, Iraji A, Salmanpour M, Ghoran SH, et al. Effects of Citrullus colocynthis L. in a rat model of diabetic neuropathy. Journal of Integrative Medicine. 2020; 18(1): 59-67.

[19]Calvo-Romero JM, Ramiro-Lozano JM. Vitamin D Levels in Patients With Type 2 Diabetes Mellitus. Journal of Investigative Medicine. 2015; 63(8):921-3.

[20]Saif-Elnasr M, Ibrahim IM, Alkady MM. Role of Vitamin D on glycemic control and oxidative stress in type 2 diabetes mellitus. Journal of Research in Medical Sciences: the official journal of Isfahan University of Medical Sciences. 2017; 22:22.

[21]Rahnavard Z, Eybpoosh S, Homami MR, Meybodi HA, Azemati B, Heshmat R, Larijani B. Vitamin D deficiency in healthy male population: Results of the Iranian multicenter osteoporosis study. Iranian Journal of Public Health. 2010; 39(3):45-52.

[22]Aarsand AK, Carlsen SM. Folate administration reduces circulating homocysteine levels in NIDDM patients on long-term metformin treatment. Journal of Internal Medicine-Oxford. 1998; 244:169-74. [23]Hoffman M. Hypothesis: hyperhomocysteinemia is an indicator of oxidant stress. Medical Hypotheses. 2011; 77(6):1088-93.

[24]Au-Yeung KKW, Woo CWH, Sung FL, Yip JCW, Siow YL, O K. Hyperhomocysteinemia activates nuclear factor $-\kappa \mathrm{B}$ in endothelial cells via oxidative stress. Circulation Research. 2004; 94(1):2836.

[25]Kibirige D, Mwebaze R. Vitamin B12 deficiency among patients with diabetes mellitus: is routine screening and supplementation justified? Journal of Diabetes \& Metabolic Disorders. 2013; 12(1):17.

[26]Qureshi SA, Ainsworth A, Winocour PH. Metformin therapy and assessment for vitamin B12 deficiency: is it necessary? Practical Diabetes. 2011; 28(7):302-4.

[27]Akinlade KS, Agbebaku SO, Rahamon SK, Balogun WO. Vitamin B12 levels in patients with type 2 diabetes mellitus on metformin. Annals of Ibadan Postgraduate Medicine. 2015; 13(2):79-83.

[28]Day C. ADA-EASD diabetes guidance: individualised treatment of hyperglycaemia. The British Journal of Diabetes \& Vascular Disease. 2012; 12(3):146-51.

[29]Satyanarayana A, Balakrishna N, Pitla S, Reddy PY, Mudili S, Lopamudra P, et al. Status of B-vitamins and homocysteine in diabetic retinopathy: association with vitamin-B12 deficiency and hyperhomocysteinemia. PloS One. 2011; 6(11):e26747-e.

[30]Krajcovicová-Kudlácková M, Blazícek P. Nutritional determinants of homocysteinemia. Casopis lekaru ceskych. 2002; 141(13):417-20. 